\title{
Avaliação dos exames de rotina no pré-natal - parte 2
}

\author{
Evaluation of the main prenatal routine exams - part 2
}

Revisão

Palavras-chave

Cuidado pré-natal

Medicina baseada em evidências

Gravidez

Bem-estar materno

Assistência perinatal

Qualidade da assistência à saúde

Keywords

Prenatal care Evidence-based medicine

Pregnancy

Maternal welfare

Perinatal assistance Quality of health care

\section{Resumo}

Uma assistência pré-natal de qualidade é essencial para garantir a saúde materna e perinatal. Atualmente, com a evolução dos métodos diagnósticos e a mudança no panorama das doenças, como o aumento da prevalência de diabetes e das doenças sexualmente transmissíveis, vários procedimentos diagnósticos estão disponíveis. Isto acarreta dificuldade para os clínicos tanto para selecionar os procedimentos mais adequados como para definir a época de aplicá-los durante a gestação, permitindo os melhores resultados para a mãe e para a criança. Esta revisão teve como objetivo avaliar os principais exames de rotina pré-natal, considerando as melhores evidências científicas disponíveis correntemente.

\section{Abstract}

A good quality prenatal assistance is essential to warrant perinatal and maternal health. Nowadays, due to the evolution of diagnostic methods and the change in illness prevalence, such as the increase in diabetes and sexually transmitted diseases, several propedeutic procedures are available. This introduces further difficulty for clinicians to select the most adequate procedures and when to apply them during gestation, assuring the best results for both mother and infant. The present review aimed at evaluating the main prenatal routine tests on the basis of the best scientific evidence presently available.
Correspondência:

Rua dos Coelhos, 300

CEP 50070-550 - Recife (PE), Brasi Fone: (083) 3321-2695/Fax: (081) 3221-0681 E-mail:melamorim@uol.com.br

Recebido

8/7/09
Centro de Atenção a Mulher do Instituto de Medicina Integral Professor Fernando Figueira - IMIP - Recife (PE), Brasil.

' Professora da Pós-graduação em Saúde Materno-Infantil do Instituto de Medicina Integral Professor Fernando Figueira - IMIP - Recife (PE), Brasil; Pesquisadora do Instituto de Pesquisa Professor Amorim Neto - IPESQ - Campina Grande (PB), Brasil.

2 Pós-graduanda (Doutorado) em Tocoginecologia pela Universidade Estadual de Campinas - UNICAMP - Campinas (SP), Brasil; Pesquisadora do Instituto de Pesquisa Professor Amorim Neto - IPESQ - Campina Grande (PB), Brasil. 


\section{Avaliação baseada em evidências}

A finalidade principal da assistência pré-natal é garantir a saúde da mãe e do feto durante toda a gravidez e o parto, identificando situações que possam aumentar o risco de desfechos desfavoráveis. A abordagem de cada gestante deve ser baseada no risco gestacional, nas características da população rastreada, na prevalência das doenças mais comuns e na avaliação das evidências disponíveis. Desta forma, realizou-se esta revisão com o objetivo de descrever os principais exames de rotina pré-natal, analisando-se as melhores evidências científicas correntemente disponíveis categorizadas em níveis. Foram pesquisados os bancos de dados MedLine/PubMed, LILACS/SciELO, a Biblioteca Cochrane, empregando o descritor "cuidado pré-natal". Utilizaram-se ainda descritores específicos para exames realizados durante a gravidez: "diabetes gestacional", "programas de rastreamento", "ultrassonografia”, "ultrassonografia Doppler", "sofrimento fetal”, "amniocentese”, "medição da translucência nucal", "pré-eclâmpsia", "cardiotocografia".

Inicialmente, pesquisaram-se os ensaios clínicos randomizados e as revisões sistemáticas, identificando-se ainda as diretrizes relacionadas aos exames pré-natais de rotina de sociedades internacionais e do Ministério da Saúde do Brasil. $\mathrm{Na}$ ausência de ensaios clínicos randomizados e metanálises específicos para uma recomendação, consideraram-se os estudos observacionais bem conduzidos e, casos estes fossem inexistentes, as recomendações das diretrizes de sociedades.

\section{Grau de recomendação e nível de evidência}

Para classificar os estudos, foram adotados o grau de recomendação e o nível de evidência, conforme sugerido pela Federação Brasileira de Ginecologia e Obstetrícia (FEBRASGO) (D) ${ }^{1}$ :

- A: estudos experimentais ou observacionais de melhor consistência;

- B: estudos experimentais ou observacionais de menor consistência;

- C: relatos ou série de casos (estudos de consistência não controlada);

Quadro 1 - Principais fatores de risco para o diabetes mellitus gestacional.

História prévia de diabetes gestaciona

Diabetes na família com parentesco em primeiro grau

Baixa estatura $(<1,50 \mathrm{~m})$

Idade superior a 25 anos

Obesidade ou ganho de peso durante a gestação

Sindrome do ovário policístico

Antecedentes obstétricos de macrossomia ou morte fetal ou neonatal

Hipertensão ou pré-eclâmpsia na gravidez atual, crescimento fetal excessivo e poli-hidrâmnio

Fonte: Ministério da Saúde (D) ${ }^{11}$.
- D: opinião desprovida de avaliação crítica, baseada em consensos, estudos fisiológicos ou de modelos animais.

\section{Rastreamento e diagnóstico de diabetes gestacional}

$\mathrm{O}$ diabetes mellitus (DM) é uma doença caracterizada principalmente por alterações no metabolismo dos carboidratos. Quando ocorre durante a gestação, recebe o nome de diabetes mellitus gestacional (DMG), definido como qualquer grau de intolerância à glicose com início ou primeiro reconhecimento na gravidez $(D)^{2,3}$. Segundo a American Diabetes Association (ADA), uma parcela significativa, ainda não mensurada, das mulheres gestam com DM tipo 2 (DMT2) não diagnosticado e, em outra parcela considerável, a gestação parece ser o gatilho para o desenvolvimento do DMT2 (D) ${ }^{2}$. Uma parcela aparentemente bem menor inicia quadro de DM tipo 1 (DMT1) durante ou logo após a gestação $(\mathrm{B})^{4}$.

A presença de diabetes na gestação aumenta o risco de danos fetais, variando de acordo com o grau de hiperglicemia e a época em que o distúrbio metabólico ocorre. Quando a hiperglicemia ocorre durante o período de embriogênese, pode determinar malformações congênitas graves, como síndrome de regressão caudal. Quando a hiperglicemia se instala na fase tardia da gestação, as complicações são representadas principalmente por macrossomia e hipoglicemia (B) ${ }^{5,6}$. Como consequência imediata da macrossomia, destaca-se o aumento da prevalência de operação cesariana e de tocotraumatismo $(B)^{7,8}$ e, como repercussão tardia, enfatiza-se o maior risco de desenvolver doenças crônicas na infância e na vida adulta jovem (obesidade, diabetes, doença cardiovascular, entre outras) (B) ${ }^{9,10}$.

Até o momento, não existem evidências para demonstrar que o rastreamento universal e o tratamento com a finalidade de reduzir os níveis glicêmicos materno diminuem a morbimortalidade materna e perinatal. Uma revisão sistemática realizada concluiu que as evidências atuais sugerem que o rastreamento universal não deve ser indicado, e sim o rastreamento seletivo, baseado nos fatores de risco (Quadro 1) ${ }^{11}$. Os autores sugerem que gestantes com intolerância discreta à glicose, cujos fetos não estão em risco, tornam-se ansiosas diante de um resultado considerado anormal e que o rastreamento não deve ser universal, mas antes baseado na presença de fatores de risco, como idade e sobrepeso $(\mathrm{A})^{12}$.

Uma força tarefa americana foi instituída em 2008 para avaliar o rastreamento de diabetes gestacional. Os autores concluíram que não existem evidências suficientes para recomendar o rastreamento universal de diabetes gestacional $(\mathrm{D})^{13}$. 
Entretanto, alguns estudos defendem o rastreamento universal como ferramenta para realizar o diagnóstico precoce, reduzindo os riscos fetais. Estudo prospectivo envolveu 1.702 gestantes não selecionadas e realizou o rastreamento para diabetes gestacional. Em primigestas, a prevalência de DMG foi similar na presença $(4,2 \%)$ ou ausência $(4,0 \%)$ de fatores de risco e, em multigestas, a prevalência de DMG aumentou na presença de fatores de risco $(8,6 \%)$ em relação à ausência $(3,9 \%)$. Os autores sugerem o rastreamento de DMG em todas gestantes entre a $24^{\mathrm{a}}$ e a $28^{\mathrm{a}}$ semanas e especial atenção para multigestas com fatores de risco $(\mathrm{B})^{14}$. Outro estudo prospectivo comparou um programa de rastreamento baseado na presença de fatores de risco ao rastreamento universal de diabetes. A prevalência de diabetes gestacional detectada no grupo que foi submetido ao rastreamento universal foi de $2,7 \%$ e foi de $1,4 \%$ no grupo que apresentava fator de risco $(\mathrm{p}<0,03)$. O rastreamento universal proporcionou um diagnóstico mais precoce em relação ao grupo rastreado com base nos fatores de risco (idade gestacional média $30 \pm 2,6$ semanas versus 33 $\pm 3,7$ semanas; $\mathrm{p}<0,05)(\mathrm{B})^{15}$.

No Brasil, o Ministério da Saúde recomenda o rastreamento universal do diabetes gestacional com a avaliação da glicemia de jejum na primeira consulta e na $20^{a}$ semana, utilizando-se de taxas $\geq 85 \mathrm{mg} / \mathrm{dL}$ como parâmetro para o emprego do teste de tolerância à glicose. Nos casos de pacientes de alto risco clínico, recomenda a repetição desta glicemia após a $24^{\mathrm{a}}$ semana de gestação (D) ${ }^{11}$.

O diagnóstico do Diabetes Gestacional é realizado com o uso do teste oral de tolerância à glicose (TOTG), sendo a glicemia dosada antes (em jejum) e depois (uma, duas e três horas) da administração de sobrecarga de glicose. Entretanto, os critérios para o diagnóstico do DMG também geram controvérsias, pois não existe um consenso sobre a sobrecarga de glicose que deve ser utilizada - Estados Unidos e Brasil utilizam sobrecarga de $100 \mathrm{~g}$; a Europa, de $75 \mathrm{~g}(\mathrm{D})^{14,15}$. Não existe também um consenso em relação a que critérios diagnósticos devem ser utilizados para interpretação da curva glicêmica. $\mathrm{O}$ National Diabetes Data Group (NDDG) e o Ministério da Saúde do Brasil propõem valores mais elevados em relação aos valores propostos por Carpenter e Coustan (B) ${ }^{16,17}$ (Quadro 2).

\section{Pesquisa de malformações fetais e doenças genéticas}

\section{Métodos não invasivos}

Ultrassonografia: translucência nucal (11므 e $13^{\underline{a}}$ semanas)

A translucência nucal (TN) vem sendo amplamente utilizada desde a década de 1990, quando Kagan et al. ${ }^{18}$ evidenciaram que o aumento da espessura cutânea presente
Quadro 2 - Parâmetros adotados para o diagnóstico de DMG segundo o NDDG e Carpenter e Coustan

\begin{tabular}{|lcc|}
\hline & $\begin{array}{c}\text { National Diabetes Data Group } \\
\mathrm{mg} / \mathrm{dL}\end{array}$ & $\begin{array}{c}\text { Carpenter e Coustan } \\
\mathrm{mg} / \mathrm{dL}\end{array}$ \\
\hline Jejum & 105 & 95 \\
1 hora & 190 & 180 \\
2 horas & 165 & 155 \\
3 horas & 145 & 140 \\
\hline
\end{tabular}

Quadro 3 - Critérios de diagnóstico adotados pelo Ministério da Saúde do Brasil

\begin{tabular}{|c|c|c|c|}
\hline \multicolumn{2}{|c|}{ Classificação } & Glicemia de jejum & $\begin{array}{l}\text { Glicemia } 2 \text { horas após } \\
\text { TTG-75 g (em mg/dL) }\end{array}$ \\
\hline \multicolumn{2}{|l|}{ Normal } & $<110$ & $<140$ \\
\hline \multirow{2}{*}{$\begin{array}{l}\text { Regulação } \\
\text { glicêmica } \\
\text { alterada }\end{array}$} & $\begin{array}{l}\text { Glicemia de } \\
\text { jejum }\end{array}$ & 110 a 125 & \\
\hline & $\begin{array}{l}\text { Tolerância } \\
\text { à glicose } \\
\text { diminuida }\end{array}$ & & 140-199 \\
\hline \multicolumn{2}{|c|}{ Diabetes mellitus } & $\geq 126$ & $\geq \underline{200}$ \\
\hline
\end{tabular}

Fonte: Ministério da Saúde (D) ${ }^{11}$.

nos indivíduos com Síndrome de Down podia ser visualizado no primeiro trimestre (entre $11^{\mathrm{a}}$ e $13^{\mathrm{a}}$ semanas e 6 dias) (B). De acordo com os primeiros trabalhos, a medida da TN associada à idade materna identifica cerca de $75 \%$ dos casos de trissomia do cromossomo 21. Quando associada à dosagem das concentrações séricas maternas da fração livre do $\beta$-hCG e da proteína plasmática $A$ associada à gestação (PAPP-A), a taxa de detecção sobe para 85 e até 90\%. A inclusão da avaliação do osso nasal (presente ou ausente) aumenta a taxa de detecção para $95 \%(B)^{18}$. Novos marcadores de primeiro trimestre, como o ângulo facial e a regurgitação da válvula tricúspide, vêm sendo acrescentados com o objetivo de melhorar a taxa de detecção e reduzir os falso-positivos e negativos (B) ${ }^{19,20}$. Outro argumento em favor da realização da avaliação da TN é que, se esta se encontra alterada, mesmo nos casos de cariótipo normal, pode contribuir para a detecção de malformações cardíacas, esqueléticas e síndromes genéticas $(\mathrm{B})^{21}$.

Nos Estados Unidos, em 2004, o National Institute of Child Health and Human Development (NICHD), a Society for Maternal-Fetal Medicine (SMFM) e o American College of Obstetricians and Gynecologists (ACOG) concluíram que o rastreamento de Síndrome de Down, no primeiro trimestre, mostrou-se eficiente, com taxa de detecção de 84\% (IC95\%=80-87\%). Sugeriram, então, que as evidências já eram suficientes para implantar o rastreamento na prática obstétrica, recomendando, no entanto, treinamento e qualificação tanto para o rastreamento ultrassonográfico e laboratorial como para a coleta de vilo corial, precedendo à implantação do rastreamento $(\mathrm{B})^{22}$.

Alguns pontos merecem ser considerados antes da implantação do rastreamento da síndrome de Down: a 
qualificação da equipe responsável pelo rastreamento, a necessidade de complementar o exame com pesquisa de cariótipo fetal nos casos de TN aumentada, a implicação psicológica do teste positivo e o impacto no nascimento de portadores da síndrome $(B)^{22}$. Assim, deve-se sempre considerar a disponibilidade de recursos e o desejo dos pais de realizar o exame (grau de recomendação B).

Ultrassonografia morfológica (entre $20^{\underline{a}}$ e $24^{\underline{a}}$ semanas)

A realização de ultrassonografia (USG) em gestantes de baixo risco tem gerado controvérsias, pois não existem evidências de que melhore o prognóstico perinatal, além da grande variação da sensibilidade do método. Uma revisão incluindo 36 estudos que envolveram 900.000 fetos com objetivo de avaliar a USG como método para detectar malformações encontrou sensibilidade média de $40,4 \%$, variando de 13,3 a $82,4 \%$ (D) ${ }^{23}$. Motivados pelas controvérsias, pesquisadores dos Estados Unidos e da Europa desenvolveram dois grandes estudos (Radius e Eurofetus), sendo o primeiro um ensaio clínico randomizado envolvendo 15.281 gestantes de baixo risco e o segundo um estudo prospectivo envolvendo 170.800 gestantes $(\mathrm{A})^{24,25}$.

No estudo Radius, um grupo de gestantes foi submetido a duas USG (entre a $15^{\mathrm{a}}$ e a $22^{\mathrm{a}}$ e entre a $31^{\mathrm{a}} \mathrm{e}$ a $35^{a}$ semanas), enquanto em um segundo grupo só se realizava USG em caso de indicação clínica. A frequência de malformações congênitas maiores foi de $2,3 \%$, e a taxa de detecção observada foi de $35 \%$ no grupo de intervenção e de $11 \%$ no grupo controle. Os autores observaram diferença na taxa de detecção de acordo com o local da realização do exame, sendo de $35 \%$ nos centros terciários e de $13 \%$ nos demais serviços. Apesar da diferença na taxa de detecção, o estudo não evidenciou impacto significante na frequência de abortamento por malformações congênitas e na taxa de sobrevida de RN com malformações potencialmente tratáveis $(\mathrm{A})^{24}$.

No estudo Eurofetus, as gestantes eram estimuladas a realizar uma USG entre a $18^{\mathrm{a}}$ e a $22^{\mathrm{a}}$ semanas, sendo realizados, em média, três exames. A taxa de detecção foi de $56,2 \%$, sendo de $73,7 \%$ para as malformações maiores e de $45,7 \%$ para as menores. Uma das justificativas para a maior taxa de detecção deste estudo, quando comparada ao Radius, foi o fato de ter envolvido apenas centros terciários $(\mathrm{A})^{25}$.

Outro ponto a ser discutido é o período ideal para realização da USG em gestantes de baixo risco. Um estudo comparando a taxa de detecção de malformações quando a USG era realizada com 18, 20 e 22 semanas não encontrou diferenças significantes. Entretanto quando o exame era realizado na $18^{\mathrm{a}}$ semana, o risco de necessidade de repetição aumentava. Os autores concluem que o período ideal para detecção de malformações encontra-se entre a $20^{\mathrm{a}}$ e a $22^{\mathrm{a}}$ semanas $(\mathrm{A})^{26}$.

Portanto, apesar de aumentar a taxa de detecção das malformações congênitas, não existem evidências de que a USG em gestantes de baixo risco melhore o prognóstico perinatal.

\section{Métodos invasivos}

Biópsia de vilo corial, amniocentese e cordocentese

A despeito da larga utilização da USG e do fato de ser este o método de escolha para a detecção de alterações estruturais, o procedimento não fornece informações sobre a constituição genética do feto. Assim, o rastreamento não invasivo (USG associada ou não aos marcadores bioquímicos), frequentemente utilizado para avaliar o risco de síndrome de Down, não permite o diagnóstico - o diagnóstico definitivo é realizado apenas por meio da análise das células fetais.

A coleta das células fetais pode ser realizada pela biópsia de vilo corial (BVC), amniocentese e cordocentese. Atualmente, o uso da cordocentese com objetivo de estudar o cariótipo fetal não é mais realizado, devido ao maior risco para o feto e à maior complexidade do procedimento quando comparado à amniocentese e à BVC. O risco de perda fetal é em torno de $1 \%$ na amniocentese clássica e de $2 \%$ na BVC $(\mathrm{B})^{27}$.

Uma revisão sistemática publicada na Cochrane envolvendo 16 ensaios clínicos randomizados concluiu que, em população de baixo risco, a amniocentese aumenta o risco de aborto espontâneo para 2,1\% quando comparado à ausência de procedimentos (1,3\%). Portanto, os pais devem ser informados sobre os riscos antes da escolha do procedimento. Sugerem ainda que, preferencialmente, a amniocentese deve ser realizada após a $16^{a}$ semana e, caso a pesquisa seja realizada em idade gestacional inferior a 15 semanas, a BVC está indicada $(\mathrm{A})^{28}$.

\section{Predição e identificação da pré-eclâmpsia}

\section{Marcadores bioquímicos}

Sempre com o intuito de identificar o mais precocemente possível as gestantes com maior risco de desenvolver pré-eclâmpsia, várias pesquisas vêm sendo realizadas com marcadores bioquímicos. Dentre os marcadores pesquisados, destacam-se: fibronectina, alfa-fetoproteína, gonadotrofina coriônica humana, PAPP-A, glicoproteína da placenta, inibina A e ativina A, sFlt1 (Soluble fms-like tyrosine kinase-1), PIGF (fator de crescimento placentário) e endoglina solúvel e proteína placentária 13 (PP13). Entretanto, novos estudos com estes marcadores precisam ser realizados com objetivo de avaliar a relevância clínica de cada teste, não existindo, até o momento, evidências que justifiquem seu uso na prática clínica diária (D ${ }^{29}$. 
Doplervelocimetria das artérias uterinas

Dentre os marcadores para pré-eclâmpsia, os métodos envolvendo o efeito Doppler são certamente os mais estudados, mas permanecem ainda como fonte de controvérsias. O aumento da resistência das artérias uterinas, representada pela presença de incisura protodiastólica e pela elevação dos índices para uma determinada idade gestacional (índice de pulsatilidade, índice de resistência (IR) e relação sístole/diástole), traduz uma falha no processo de placentação, com aumento do risco de restrição de crescimento intrauterino e pré-eclâmpsia $(\mathrm{A})^{30}$. Uma revisão sistemática que incluiu 27 estudos observacionais concluiu que a doplervelocimetria da artéria uterina tem valor limitado como teste preditivo de pré-eclampsia em gestantes de baixo risco $(A)^{31}$.

Em contrapartida, outros estudos sugerem que a doplervelocimetria em mulheres de risco pode ser um método útil para predizer a pré-eclâmpsia e a restrição de crescimento fetal (RCF). Um estudo prospectivo envolvendo 52 gestantes com fatores de risco para pré-eclâmpsia avaliou a sensibilidade do método. Os autores verificaram que a sensibilidade da presença de incisura para predizer pré-eclâmpsia, RCF e complicações severas na gravidez foi de 75, 71 e 69\%, respectivamente. A sensibilidade do IR >0,58 para predizer RCF, pré-eclâmpsia, morte intrauterina e complicações graves foi de 67, 50, 75 e $80 \%$, respectivamente $(\mathrm{B})^{32}$.

A avaliação das artérias uterinas também vem sendo realizada no primeiro trimestre, baseada no conceito de que o desaparecimento da incisura protodiastólica precoce teria um efeito protetor. Quanto mais precocemente for evidenciado o seu desaparecimento, maior será o efeito protetor. Entretanto, a realização de uma única medida doplervelocimétrica, de forma precoce, em uma população não selecionada, apresenta valor clínico limitado. Essa avaliação precoce deve ser reservada às mulheres com risco elevado de desenvolver pré-eclâmpsia e também deve ser repetida ou associada a outras formas de predição para se obter maior acurácia $(\mathrm{B})^{33}$.

Atualmente, os estudos procuram agregar testes para aumentar a sensibilidade dos métodos. Em estudo prospectivo avaliando 348 nulíparas entre a $11^{\mathrm{a}}$ e a $14^{\mathrm{a}}$ semanas, além da doplervelocimetria das artérias uterinas, era calculado o volume placentário por meio de USG tridimensional. O volume placentário foi significativamente menor entre as mulheres que posteriormente desenvolveram pré-eclâmpsia e, de forma ainda mais significativa, entre aquelas em que a gestação foi interrompida antes da $32^{a}$ semana. A sensibilidade, tanto do IP quanto do volume placentário para predição da pré-eclâmpsia, foi de 50 e $56 \%$, respectivamente, e de 66,7 e 66,7\%, quando se considerou a pré-eclâmpsia precoce. A combinação do IP das artérias uterinas com o volume placentário aumentou para $68,7 \%$ a sensibilidade para predição de pré-eclâmpsia e para $83,3 \%$ quando foram considerados os casos de pré-eclâmpsia precoce $(A)^{34}$.

Um estudo envolvendo 3.359 gestantes encontrou uma prevalência de pré-eclâmpsia de $3 \%$, sendo $0,7 \%$ de início precoce (antes da $34^{\mathrm{a}}$ semana) e 2,3\% tardia (início após a $34^{\mathrm{a}}$ semana). Após análise de regressão múltipla, o índice de pulsatilidade das artérias uterinas e a média da pressão arterial entre a $22^{\mathrm{a}}$ e a $24^{\mathrm{a}}$ semanas foram fatores independentes para a predição de pré-eclâmpsia. A taxa de falsos-positivos foi de $10 \%$ e a taxa de detecção foi de $100 \%$ para pré-eclâmpsia precoce e de $56,4 \%$ para a $\operatorname{tardia}(\mathrm{B})^{35}$.

Em recente revisão sistemática incluindo 27 estudos, o National Institute for Health Research (NIHR) concluiu que os principais métodos preditores da préeclâmpsia são: índice de massa corpórea $>34 \mathrm{~kg} / \mathrm{m}^{2}$, níveis elevados de alfa-fetoproteína plasmática e incisura protodiastólica bilateral na artéria uterina. Os autores concluíram que nenhum dos testes avaliados apresenta acurácia suficiente para incorporação de rotina à prática clínica diária $(\mathrm{A})^{36}$

Baseados nas evidencias atuais, a Sociedade de Ginecologia e Obstetrícia do Canadá, o Colégio Americano de Ginecologia e Obstetrícia (ACOG), o Royal College of Obstetricians and Gynaecologists (RCOG) e o Ministério da Saúde do Brasil não recomendam o uso rotineiro da doplervelocimetria em gestantes de baixo risco (D) ${ }^{11,37-39}$.

\section{Avaliação do crescimento e do bem-estar fetal}

\section{Avaliação do crescimento fetal}

A USG realizada após a $24^{\mathrm{a}}$ semana é controversa em gestantes de baixo risco. Em uma revisão sistemática de nove ensaios clínicos, os autores observaram que a USG de rotina está associada à detecção precoce de gestação múltipla $(\mathrm{OR}=0,08$; IC95\%=0,52-0,72) e redução na taxa de indução do parto devido à gestação pós-termo $(\mathrm{OR}=0,61$; IC95\%=0,52-0,72). Não foi observada diferença significativa na mortalidade perinatal $(\mathrm{OR}=0,86$; IC95\% $\%$ 0,67-1,12) $(\mathrm{A})^{40}$.

Segundo o American College of Obstetricians and Gynecologists (ACOG), a USG é um método eficiente para determinar a idade gestacional, o número de fetos, a viabilidade e a implantação da placenta e a precisão para determinação da idade gestacional é maior quando o exame é efetuado na primeira metade da gestação. Em relação à avaliação do crescimento fetal, a USG de rotina, quando realizada em um único momento, não é eficaz, devendo ser seriada, com intervalos entre duas e quatro semanas e baseada em indicações específicas (D) ${ }^{41}$.

No Brasil, devido à falta de evidências para demonstrar melhora dos resultados perinatais, a realização da USG em gestantes de baixo risco vai depender da disponibilidade 
do serviço. Não há recomendação para avaliar rotineiramente o crescimento fetal neste grupo de gestantes. Em gestantes de alto risco, a avaliação do crescimento e da vitalidade fetal é recomendada $(\mathrm{D})^{11}$.

\section{Avaliação do bem-estar fetal}

A avaliação da vitalidade fetal em gestantes de alto risco tem como objetivo principal assegurar o bem-estar do feto. Dentre os procedimentos utilizados para avaliar o bem-estar fetal, destacam-se o perfil biofísico fetal (PBF), a cardiotocografia (CTG) e a doplervelocimetria.

A utilização clínica do PBF baseia-se na hipótese de que, em razão da redução dos níveis de oxigênio, o feto reduz os movimentos com o objetivo de diminuir o consumo. Uma revisão sistemática analisou cinco ensaios clínicos envolvendo 2.974 gestantes de alto risco. Não foram observadas diferenças significativas em relação à mortalidade perinatal ( $\mathrm{RR}=1,33$; IC95\%=0,60-2,98) ou ao Apgar menor que sete no quinto minuto $(\mathrm{RR}=1,27$; IC95\% $=0,85-1,92)$. Observou-se, ainda, analisando os resultados de dois ensaios clínicos ( $\mathrm{n}=280)$, um aumento do risco de cesárea no grupo monitorizado por meio do PBF ( $R R=1,60$; IC95\% $=1,05$ $2,44)$. Os autores concluíram que não existem evidências suficientes para recomendar o uso de PBF na avaliação do bem-estar fetal em gestantes de alto risco $(\mathrm{A})^{42}$. Apesar da ausência de evidências para recomendar o uso de PBF, inclusive em gestações de alto risco, o ACOG e o RCOG aconselham a inclusão do PBF na monitorização de fetos com restrição de crescimento intrauterino (D) ${ }^{38,39}$.

Não obstante a CTG ser um dos exames mais empregados na avaliação da vitalidade fetal, também não existem evidências para recomendar o seu uso mesmo em gestantes de alto risco. Uma revisão sistemática envolvendo 1.588 gestantes de baixo e alto risco foi realizada com o objetivo de avaliar o efeito da CTG durante o período antenatal nos escores de Apgar e na taxa de cesárea. Os autores concluíram que a CTG antenatal não determinou efeito significante na morbimortalidade perinatal e, ao contrário, observou-se uma tendência ao aumento de morte perinatal no grupo submetido à CTG $(\mathrm{OR}=2,85 ; \mathrm{IC} 95 \%=0,99-7,12)$. Não se observou aumento na incidência de procedimentos, como cesárea ou indução do parto $(\mathrm{A})^{43}$. O ACOG e o RCOG não recomendam o uso rotineiro da CTG, restringindo sua utilização aos casos de RCF (D) $)^{38,39}$.

Em relação à doplervelocimetria, duas revisões sistemáticas avaliando o uso da artéria umbilical na avaliação do bem-estar fetal concluíram que, em mulheres com pré-eclâmpsia e em fetos com restrição de crescimento, a doplervelocimetria reduz significativamente a mortalidade perinatal e as intervenções obstétricas desnecessárias. A revisão sistemática publicada pelo grupo Cochrane, avaliando 11 ensaios clínicos envolvendo 7.000 gestantes, concluiu que o emprego do Doppler em gestantes de alto risco estava associado a uma tendência à redução da mortalidade perinatal $(\mathrm{OR}=0,71$; IC95\%=0,50-1,01). Estes achados foram obtidos principalmente quando os estudos envolviam gestantes hipertensas ou fetos apresentando restrição de crescimento. Os autores observaram ainda uma redução na indução do parto $(\mathrm{OR}=0,83$; IC95\% $=0,74-0,93)$ e na admissão hospitalar $(\mathrm{OR}=0,56$; IC95\% $=0,43-0,72)(\mathrm{A})^{44}$.

Com base nas evidências atuais, a Sociedade de Ginecologia e Obstetrícia do Canadá, o ACOG, o RCOG e o Ministério da Saúde do Brasil recomendam a realização da doplervelocimetria em gestantes com suspeita de insuficiência placentária (D) $)^{11,37-39}$.

\section{Conclusões}

Apesar de exisitir recomendação do Ministério da Saúde para o rastreamento universal de DMG, ainda não existe consenso se o rastreamento deve ser universal ou baseado em fatores de risco. As evidências são insuficientes para se fazer recomendação, devendo cada país ou região fazê-lo com base nas características e expectativas da população a ser rastreada.

A ultrassonografia de rotina não melhora o prognóstico perinatal isoladamente em gestações de baixo risco. Entretanto, uma ultrassonografia precoce tem a vantagem de permitir o diagnóstico oportuno das gestações múltiplas e a datação mais acurada da idade gestacional, reduzindo desta forma o número de induções por gestação prolongada. Não há evidências apoiando sua indicação rotineira com o propósito de melhorar o prognóstico perinatal. A decisão de incorporar ou não o exame à prática obstétrica deve considerar recursos disponíveis, qualidade dos serviços de Saúde e características e expectativas dos casais.

Não há evidências comprovando que a avaliação da vitalidade fetal, com o emprego de doplervelocimetria, cardiotocografia e perfil biofísico fetal, em gestantes de baixo risco, melhore os resultados perinatais. Esses exames possuem acurácia limitada em grupos de gestantes de baixo risco, e sua realização pode levar a falsos-positivos, acarretando angústia materna e excesso de intervenções. Não são, portanto, recomendados como rotina em gestantes de baixo risco. Entretanto, em gestantes de alto risco, existem evidências de que a doplervelocimetria melhora os resultados perinatais e reduz o risco de indução de parto e cesárea.

O rastreamento genético deve ser realizado somente após esclarecimento da gestante/do casal, dependendo fundamentalmente de características da(s) paciente(s) e da população; prevalência das condições sob pesquisa; benefícios decorrentes do diagnóstico e do tratamento da condição pesquisada; evidências científicas documentadas sobre a acurácia dos testes; expectativas da(s) paciente(s) e recursos disponíveis. Até o presente momento, as evidências são insuficientes para recomendar a sua utilização de rotina em população de baixo risco. 
1. Associação Médica Brasileira. Conselho Federal de Medicina [Internet]. Projeto Diretrizes: introdução. [citado 2009 Maio 6]. Disponível em: http://www.projetodiretrizes.org.br/projeto_ diretrizes/texto_introdutorio.pdf

2. American Diabetes Association. Diagnosis and classification of diabetes mellitus. Diabetes Care. 2008;31 Suppl 1:S55-60.

3. Alberti KGMM, Zimmet PZ. Definition, diagnosis and classification of diabetes mellitus and its complications. Part 1: Diagnosis and classification of diabetes mellitus. Report of a WHO Consultation. Geneva: WHO; 1999.

4. Schmidt MI, Duncan BB, Reichelt AJ, Branchtein L, Matos MC Costa e Forti $A$, et al. Gestational diabetes mellitus diagnosed with a 2-h 75-g oral glucose tolerance test and adverse pregnancy outcomes. Diabetes Care. 2001;24(7):1151-5.

5. Macintosh MC, Fleming KM, Bailey JA, Doyle P, Modder J, Acolet $D$, et al. Perinatal mortality and congenital anomalies in babies of women with type 1 or type 2 diabetes in England, Wales, and Northern Ireland: population based study. BM. 2006;333(7560): 177.

6. Confidential Enquiry into Maternal and Child Health: pregnancy in women with type 1 and type 2 diabetes in 2002-03, England, Wales, and Northern Ireland. London: CEMACH; 2005.

7. Boulet SL, Alexander GR, Salihu HM, Pass M. Macrosomic births in the United States: determinants, outcomes, and proposed grades of risk. Am J Obstet Gynecol. 2003;188(5):1372-8.

8. Jolly MC, Sebire NJ, Harris JP, Regan L, Robinson S. Risk factors for macrosomia and its clinical consequences: a study of 350,31 1 pregnancies. Eur J Obstet Gynecol Reprod Biol. 2003;111(1): 9-14.

9. Boney CM, Verma A, Tucker R, Vohr BR. Metabolic syndrome in childhood: association with birth weight, maternal obesity, and gestational diabetes mellitus. Pediatrics. 2005; 115(3):e290-6.

10. Clausen T, Burski TK, Oyen N, Godang K, Bollerslev J, Henriksen T. Maternal anthropometric and metabolic factors in the first half of pregnancy and risk of neonatal macrosomia in term pregnancies. A prospective study. Eur J Endocrinol. 2005;153(6):887-94.

11. Ministério da Saúde. Secretaria de Atenção à Saúde. Departamento de Ações Programáticas Estratégicas. Pré-natal e puerpério: atenção qualificada e humanizada: manual técnico. Brasília: MS; 2006.

12. Scott DA, Loveman E, Mclntyre L, Waugh N. Screening for gestational diabetes: a systematic review and economic evaluation. Health Technol Assess. 2002;6(11):1-161.

13. US Preventive Services Task Force. Screening for gestational diabetes mellitus: US Preventive Services Task Force recommendation statement. Ann Intern Med. 2008;148(10):759-65.

14. McCarthy AD, Curciarello R, Castiglione N, Tayeldín MF, Costa $D$, Arnol V, et al. Universal versus selective screening for the detection, control and prognosis of gestational diabetes mellitus in Argentina. Acta Diabetol. In press 2009.

15. Griffin ME, Coffey M, Johnson H, Scanlon P, Foley M, Stronge $J$, et al. Universal vs. risk factor-based screening for gestational diabetes mellitus: detection rates, gestation at diagnosis and outcome. Diabet Med. 2000;17(1):26-32.

16. Reece EA, Leguizamón $G$, Wiznitzer A. Gestational diabetes: the need for a common ground. Lancet. 2009;373(9677): 1789-97.

17. Gokcel A, Bagis T, Killicadag EB, Tarim E, Guvener N. Comparison of the criteria for gestational diabetes mellitus by NDDG and
Carpenter and Coustan, and the outcomes of pregnancy. J Endocrinol Invest. 2002;25(4):357-61.

18. Kagan KO, Wright D, Valencia C, Maiz N, Nicolaides KH. Screening for trisomies 21,18 and 13 by maternal age, fetal nuchal translucency, fetal heart rate, free beta-hCG and pregnancy-associated plasma protein-A. Hum Reprod. 2008;23(9):1968-75.

19. Borenstein M, Persico N, Kagan KO, Gazzoni A, Nicolaides KH. Frontomaxillary facial angle in screening for trisomy 21 at $11+0$ to $13+6$ weeks. Ultrasound Obstet Gynecol. 2008;32(1):5-1 1 .

20. Falcon O, Aver M, Gerovassili A, Spencer K, Nicolaides KH. Screening for trisomy 21 by fetal tricuspid regurgitation, nuchal translucency and maternal serum free beta-hCG and PAPP-A at $11+0$ to $13+$ 6 weeks. Ultrasound Obstet Gynecol. 2006;27(2):151-5.

21. Atzei A, Gajewska K, Huggon IC, Allan L, Nicolaides KH. Relationship between nuchal translucency thickness and prevalence of major cardiac defects in fetuses with normal karyotype. Ultrasound Obstet Gynecol. 2005;26(2):154-7.

22. Driscoll DA, Gross SJ; Professional Practice and Guidelines Committee. First trimester diagnosis and screening for fetal aneuploidy. Genet Med. 2008; 10(1):73-5.

23. Levi S. Ultrasound in prenatal diagnosis: polemics around routine ultrasound screening for second trimester fetal malformations. Prenat Diagn. 2002;22(4):285-95.

24. Crane JP, LeFevre ML, Winborn RC, Evans JK, Ewigman BG, Bain RP, et al. A randomized trial of prenatal ultrasonographic screening: impact on the detection, management, and outcome of anomalous fetuses. The RADIUS Study Group. Am J Obstet Gynecol. 1994;171(2):392-9.

25. Grandjean $H$, Larroque D, Levi S. Sensitivity of routine ultrasound screening of pregnancies in the Eurofetus database. The Eurofetus Team. Ann N Y Acad Sci. 1998;847:1 18-24.

26. Schwärzler $P$, Senat MV, Holden D, Bernard JP, Masroor T, Ville $Y$. Feasibility of the second-trimester fetal ultrasound examination in an unselected population at 18,20 or 22 weeks of pregnancy: a randomized trial. Ultrasound Obstet Gynecol. 1999; 14(2):92-7.

27. Tabor $\mathrm{A}$, Vestergaard $\mathrm{CH}$, Lidegaard $\varnothing$. Fetal loss rate after chorionic villus sampling and amniocentesis: an 11-year national registry study. Ultrasound Obstet Gynecol. 2009;34(1):19-24.

28. Alfirevic Z, Mujezinovic F, Sundberg K. Amniocentesis and chorionic villus sampling for prenatal diagnosis. Cochrane Database Syst Rev. $2009 ;(1)$ :CD003252.

29. Conde-Agudelo A, Villar J, Lindheimer M. World Health Organization systematic review of screening tests for preeclampsia. Obstet Gynecol. 2004;104(6):1367-91.

30. Cnossen JS, Morris RK, ter Riet G, Mol BW, van der Post JA, Coomarasamy A, et al. Use of uterine artery Doppler ultrasonography to predict pre-eclampsia and intrauterine growth restriction: a systematic review and bivariable meta-analysis. CMAJ. 2008;178(6):701-11.

31. Meads CA, Cnossen JS, Meher S, Juarez-Garcia A, ter Riet $G$, Duley L, et al. Methods of prediction and prevention of preeclampsia: systematic reviews of accuracy and effectiveness literature with economic modelling. Health Technol Assess. 2008; 12(6): 1-270.

32. Axt-Fliedner R, Schwarze A, Nelles I, Altgassen C, Friedrich M, Schmidt $W$, et al. The value of uterine artery Doppler ultrasound 
in the prediction of severe complications in a risk population. Arch Gynecol Obstet. 2005;271(1):53-8.

33. Gómez O, Martínez JM, Figueras F, Del Río M, Borobio V, Puerto B, et al. Uterine artery Doppler at 1 1-14 weeks of gestation to screen for hypertensive disorders and associated complications in an unselected population. Ultrasound Obstet Gynecol. 2005;26(5):490-4.

34. Rizzo G, Capponi A, Cavicchioni O, Vendola M, Arduini D. First trimester uterine Doppler and three-dimensional ultrasound placental volume calculation in predicting pre-eclampsia. Eur J Obstet Gynecol Reprod Biol. 2008;138(2):147-51.

35. Onwudiwe N, Yu CK, Poon LC, Spiliopoulos I, Nicolaides KH. Prediction of pre-eclampsia by a combination of maternal history, uterine artery Doppler and mean arterial pressure. Ultrasound Obstet Gynecol. 2008;32(7):877-83.

36. Ilekis JV, Reddy UM, Roberts JM. Preeclampsia-a pressing problem: an executive summary of a National Institute of Child Health and Human Development workshop. Reprod Sci. 2007;14(6):508-23.

37. Gagnon R, Van den Hof M; Diagnostic Imaging Committee, Executive and Council of the Society of Obstetricians and Gynaecologists of Canada. The use of fetal Doppler in obstetrics. J Obstet Gynaecol Can. 2003;25(7):601-14.
38. American College of Obstetricians and Gynecologists. Intrauterine growth restriction [ACOG Practice Bulletin, 12]. Washington, DC: ACOG; 2000.

39. Royal College of Obstetricians and Gynaecologists. The investigation and management of the small-for-gestatational-age fetus [Guideline, 31]. London: RCOG; 2002.

40. Bricker L, Neilson JP, Dowswell T. Routine ultrasound in late pregnancy (after 24 weeks' gestation). Cochrane Database Syst Rev. 2009;(1):CD001451.

41. American College of Obstetricians and Gynecologists. ACOG Practice Bulletin No. 101: ultrasonography in pregnancy. Obstet Gynecol. 2009; $113(2 \mathrm{Pt}$ 1):45 1-61.

42. Lalor JG, Fawole B, Alfirevic Z, Devane D. Biophysical profile for fetal assessment in high risk pregnancies. Cochrane Database Syst Rev. 2008;(4):CD000038.

43. Pattison N, McCowan L. Cardiotocography for antepartum fetal assessment. Cochrane Database Syst Rev. 2008;(4): CD001068.

44. Neilson JP, Alfirevic Z. Doppler ultrasound for fetal assessment in high risk pregnancies. Cochrane Database Syst Rev. 2008;(4):CD000073. 